\title{
Incidence and risk factors of HIV-associated non-Hodgkin-lymophma in the era combined anti-retroviral therapy. A European multi-cohort study J Bohlius ${ }^{1,2}$
}

\author{
Address: ${ }^{1}$ for the Collaboration of Observational HIV Epidemiological Research Europe (COHERE) Study Group and ${ }^{2}$ Institute of Social and \\ Preventive Medicine, University of Bern, Bern, Switzerland \\ from I I th International Conference on Malignancies in AIDS and Other Acquired Immunodeficiencies (ICMAOI): Basic, Epidemiologic, and Clinical \\ Research \\ Bethesda, MD, USA. 6-7 October 2008 \\ Published: 17 June 2009 \\ Infectious Agents and Cancer 2009, 4(Suppl 2):O2 doi:I0.II86/I750-9378-4-S2-O2
}

This abstract is available from: http://www.infectagentscancer.com/content/4/S2/O2

(c) 2009 Bohlius; licensee BioMed Central Ltd.

\section{Background}

Incidence and risk factors of HIV-associated non-Hodgkin lymphoma (NHL) in the era of combined anti-retroviral therapy (CART) have not been well defined. We analyzed incidence and risk factors for HIV-related NHL in the era of cART within the framework of a large European multicohort collaborative study.

\section{Methods}

We analyzed the data of HIV-infected adult patients (over 16 years old) who were cART naïve at inclusion and started cART after January 1, 1998. cART was defined as regimen with at least three antiretroviral drugs. Patients had to have at least one CD4 cell count measurement after January 1, 1998, and before start of HAART or diagnosis of NHL. Both patients developing Primary Brain Lymphoma and systemic NHL were included in the analysis. Incidence rates were calculated based on the Poisson distribution; risk factors were estimated using crude and adjusted Weibull models, with random effects to account for heterogeneity between cohorts. Models with time varying covariates were used to explore the effect of CD4 cells counts and plasma HIV-RNA loads over time. The database included a total of 67,659 patients. Seventeen percent of patients $(11,354)$ were excluded from the analysis because they did not meet the inclusion criteria outlined above.

\section{Results}

We included 56,305 patients from 22 cohort studies across Europe. During 212,042 person-years of follow up, 583 patients developed NHL. The incidence in patients not on cART was 519 (95\% CI 448 to 602) per 100,000 person-years compared to 229 (95\% CI 208 to 252) per 100,000 person-years in those on cART. The incidence of Primary Brain Lymphoma was 56.7 (95\% CI 36 to 89) per 100,000 person-years in patients not on CART and 24 (95\% CI 18 to 33) per 100,000 person years in patients on cART. The incidence of systemic Non-Hodgkin Lymphoma other than Primary Brain Lymphoma was 463 (95\% CI 395 to 541) per 100,000 in patients not on cART and 205 (95\% CI 185 to 227) per 100,000 person years in patients on CART. In cART naïve patients the risk for NHL increased with age, high plasma HIV-RNA loads and decreasing CD4 cell counts. In patients receiving cART risk factors for developing NHL were low CD4 cell counts, higher age, belonging to the transmission risk group men having sex with men and previous diagnosis of Kaposi Sarcoma.

\section{Conclusion}

Combined anti-retroviral therapy reduces the risk of developing NHL. In the era of cART more advanced immunodeficiency is the dominant risk factor for developing NHL both in patients receiving and not receiving cART for treatment of HIV infection. 